\section{Soluble adhesion molecules in NIDDM: increased concentration, relation to glycometabolic control and possible pathophysiological significance}

\section{Dear Sir,}

Recently, Cominacini et al. [1] described increased concentrations of soluble adhesion molecules in patients suffering from insulin-dependent and non-insulin-dependent diabetes mellitus (NIDDM). Soluble E-selectin and intercellular adhesion molecule-1 (ICAM-1) concentrations were increased in NIDDM whereas vascular adhesion molecule-1 (VCAM-1) demonstrated no significant changes when compared to a control group. Interestingly, E-selectin concentrations positively correlated with glycaemic control as measured by glycated haemoglobin values.

We performed a similar study in 60 younger patients (below the age of 60 years) suffering from NIDDM to examine soluble adhesion molecules and their association with glycometabolic control and established cardiovascular risk factors [2]. Accordingly, we feel that consideration of available data could have positively influenced the discussion provided in the report of Cominacini et al. [1].

Relative to control subjects, increased levels of E-selectin and VCAM-1 were found in our study whereas ICAM-1 was unchanged. Levels of soluble adhesion molecules failed to correlate with glycaemic control (fructosamine, glycated haemoglobin) as well as with urinary albumin excretion. However, E-selectin showed a significant correlation with LDL cholesterol. The lack of significant associations between E-selectin and total cholesterol, triglycerides, HDL and LDL cholesterol in Cominacini et al's investigation [1] is probably due to the fact that their patients had lipid metabolism parameters not significantly different from the control group (except decreased HDL cholesterol). The association between disturbed lipoprotein metabolism and E-selectin can further be suspected from the finding that the patients with either diabetes or hyperlipoproteinaemia (types IIa and IIb) demonstrated increased E-selectin levels.

Corresponding author: Dr. M. Steiner, University of Rostock, Faculty of Medicine, Institute of Clinical Chemistry and Pathobiochemistry, Ernst-Heydemann-Straße 6, D-18057 Rostock, Germany
We agree that increased levels of E-selectin reflect endothelial cell activation. However, it should be mentioned that by using E-selectin alone it seems impossible to characterise the true status of the vascular endothelium in disease. A set of parameters including von Willebrand factor, soluble thrombomodulin and endothelial cell-specific soluble adhesion molecules [3] represent an appropriate tool with which to prospectively investigate vascular endothelium in diabetes and its complications. Unfortunately, to the best of our knowledge, no such study has been performed.

Finally, it is tempting to speculate on the pathophysiological significance of increased soluble adhesion molecules in the complex network of humoral and cellular factors resulting in vascular diabetic complications. Soluble endothelial adhesion molecules (E-selectin, VCAM-1) have recently been shown to mediate angiogenesis [4]. Moreover, this effect seems to be independent of the release of angiogenic cytokines by the endothelium. Vascular diabetic complications depend on neovascularisation, and angiogenic factors including vascular endothelial growth factor [5] and insulin-like growth factors I and II $[6,7]$ have been associated with the progression of diabetic retinopathy. Hence, at least two of the soluble adhesion molecules are to be added to a growing list of molecules promoting angiogenesis in a variety of diseases including diabetes [8]. Future studies on soluble adhesion molecules are required to provide answers to many unresolved questions. One of the most intriguing concerns the proposed pathogenic role of increased soluble adhesion molecules and whether they simply reflect endothelial cell activation/injury in diabetes. If the former hypothesis proves correct, soluble adhesion molecules such as E-selectin and VCAM-1 might become target molecules with which to fight vascular diabetic complications by using antiangiogenic treatment approaches.

Yours sincerely,

M.Steiner, K. M. Reinhardt, A.D. Blann

\section{References}

1. Cominacini L, Fratta Pasini A, Garbin U et al. (1995) Elevated levels of soluble E-selectin in patients with IDDM and NIDDM: relation to metabolic control. Diabetologia 38: 1122-1124

2. Steiner M, Reinhardt KM, Krammer B, Ernst B, Blann AD (1994) Increased levels of soluble adhesion molecules in type 2 (non-insulin dependent) diabetes mellitus are independent of glycaemic control. Thromb Haemost 72: 979-984 
3. Blann AD, Taberner DA (1995) A reliable marker of endothelial cell dysfunction: does it exist? $\mathrm{Br} \mathrm{J}$ Haematol 90: 244-248

4. Koch AE, Halloran MM, Haskell CJ, Shah MR, Polverini PJ (1995) Angiogenesis mediated by soluble forms of E-selectin and vascular cell adhesion molecule-1. Nature 376: 517519

5. Aiello LP, Avery RL, Arrigg PG et al. (1994) Vascular endothelial growth factor in ocular fluid of patients with diabetic retinopathy and other retinal disorders. N Engl J Med 331: $1480-1487$
6. Grant MB, Mames RN, Fitzgerald C, Ellis EA, Aboufriekha M, Guy J (1993) Insulin-like growth factor I acts as an angiogenic agent in rabbit cornea and retina: comparative studies with basic fibroblast growth factor. Diabetologia 36: 282-291

7. Meyer-Schwickerath R, Pfeiffer A, Blum WF et al. (1993) Vitreous levels of the insulin-like growth factors I and II, and the insulin-like growth factor binding proteins 2 and 3, increase in neovascular eye disease. Studies in nondiabetic and diabetic subjects. J Clin Invest 92: 2620-2625

8. Folkman J (1995) Angiogenesis in cancer, vascular, rheumatoid and other disease. Nature Med 1:27-31

\section{Response from the authors}

\section{Dear Sir,}

Dr. Steiner et al. discuss our recently published results on adhesion molecules in diabetic patients [1]. In this paper increased E-selectin concentrations were found in patients with insulin-dependent and non-insulin-dependent diabetes mellitus (NIDDM) and in a group of dyslipidaemic subjects. Intercellular adhesion molecule-1 (ICAM-1) levels were found to be elevated only in the patients with NIDDM while no differences in vascular adhesion molecule-1 (VCAM-1) concentrations were detected in the other groups of subjects. Plasma Eselectin positively correlated with the glycated haemoglobin in the diabetic patients. E-selectin, however, was not related to the concentration of plasma lipids despite the fact that it was found to be elevated in hyperlipoproteinaemic subjects.

In a previous paper Steiner et al. [2] performed a similar study in 60 patients suffering from NIDDM in order to examine soluble adhesion molecules and their association with metabolic control and cardiovascular risk factors. When compared to control subjects, elevated levels of E-selectin and VCAM-1 levels were found whereas the ICAM-1 concentration was similar. In Steiner's investigation the levels of adhesion molecules were not correlated with glycaemic control. At variance, E-selectin showed a significant correlation to LDL cholesterol.

I agree with the fact that consideration of Steiner's report [2] could have positively influenced the discussion provided in our paper. Unfortunately our investigation was planned, written and sent for publication before we could have this report to hand. Steiner's report therefore was not deliberately omitted and we were perfectly aware that other data on adhesion molecules in diabetic patients had been published previously $[3,4]$.

There are differences in the results of the two papers, but their main result, i.e. the increase of E-selectin in patients suffering from NIDDM is similar. I believe that this finding is extremely important.

Contrary to Steiner's investigation, we did not find a significant correlation between E-selectin and lipid levels and LDL cholesterol in particular. Actually, in order to exclude possible interference from plasma lipids on the plasma concentrations of E-selectin and other adhesion molecules, only the patients with lipid parameters similar to those of the control group were considered in our study. In Steiner's investigation both LDL cholesterol and triglyceride levels were significantly

Corresponding author: Dr. L. Cominacini, Istituto di Semeiotica e Nefrologia Medica, Università di Verona, Ospedale Policlinico, I-37134 Verona, Italy

higher in the diabetic than in the control group. Given the demonstrated association between increased lipid concentrations and E-selectin in dyslipidaemic patients [1] the weak association between E-selectin and LDL cholesterol concentrations found by Steiner and co-workers [2] may also reflect the augmented concentration of the lipoprotein in the diabetic patients relative to the control subjects. The fact that in our study we did not find a relationship between E-selectin and LDL cholesterol concentrations in the group of dyslipidaemic patients may also be due to the limited number of subjects studied.

Contrary to our results [1], in Steiner's investigation the levels of soluble adhesion molecules failed to correlate with glycaemic control [2]. On the basis of the already published results, of course, it is difficult to explain why we found a correlation between E-selectin concentrations and glycaemic control and Steiner and co-workers did not. It should be noted, however, that the diabetic patients studied in Steiner's investigation were not only dyslipidaemic, but $70 \%$ were also hypertensive and it has recently been demonstrated that hypertension is a pathological condition also characterized by increased levels of plasma E-selectin [5]. In these patients, therefore, there were at least two other risk factors in addition to diabetes per se that could interfere with the expression of E-selectin on endothelial cells and hence on its plasma concentration.

We believe that the only correct way to accurately demonstrate a possible relation between plasma $E$-selectin concentrations and glycaemic control is to study these parameters in the same patient before and after good glycaemic control has been achieved. It must be pointed out, however, that early glycation products, for example glycated haemoglobin, are poor indicators of irreversible advanced glycation end product formation and that only these products can induce the expression of adhesion molecules, especially E-selectin [6]. Future studies on the correlation between adhesion molecule plasma concentrations and circulating advanced glycation end products in diabetic patients are required.

Yours sincerely

L. Cominacini, A. Fratta Pasini, U. Garbin, V. Lo Cascio

\section{References}

1. Cominacini L, Fratta Pasini A, Garbin U et al. (1995) Elevated levels of soluble E-selectin in patients with IDDM and NIDDM: relation to metabolic control. Diabetologia 38: 1122-1124

2. Steiner M, Reinhardt KM, Krammer B, Ernst B, Blann AD (1994) Increased levels of soluble adhesion molecules in type 2 (non-insulin dependent) diabetes mellitus are 